\title{
MICROENCAPSULAÇÃO DO AGENTE QUELANTE SULFOXINA EM MICROESFERAS DE QUITOSANA PREPARADAS POR SPRAY DRYING COMO NOVO ADSORVENTE PARA ÍONS METÁLICOS
}

\author{
Luciano Vitali, Mauro Cesar Marghetti Laranjeira e Valfredo Tadeu de Fávere* \\ Departamento de Química, Universidade Federal Santa Catarina, CP 476, 88040-900 Florianópolis - SC, Brasil \\ Norberto Sanches Gonçalves \\ Departamento de Ciências Exatas e da Terra, Universidade Federal de São Paulo, 09972-270 Diadema - SP, Brasil
}

Recebido em 26/7/07; aceito em 14/3/08; publicado na web em 26/8/08

\begin{abstract}
MICROENCAPSULATION OF THE CHELATING AGENT SULFOXINE INTO MICROSPHERES OF CHITOSAN PREPARED BY SPRAY DRYING AS A NEW ADSORBENT FOR METALIC IONS. In this work, a new adsorbent was prepared by microencapsulation of sulfoxine into chitosan microspheres by the spray drying technique. The new adsorbent was characterized by Raman spectroscopy, scanning electron microscopy and microanalysis of energy dispersive $\mathrm{X}$-rays. The $\mathrm{Cu}(\mathrm{II})$ adsorption was studied as a function of $\mathrm{pH}$, time and concentration. The optimum $\mathrm{pH}$ was found to be 6.0. The kinetic and equilibrium data showed that the adsorption process followed the pseudo second-order kinetic model and the Langmuir isotherm model over the entire concentration range. An increase of $8.0 \%$ in the maximum adsorption capacity of the adsorbent $\left(53.8 \mathrm{mg} \mathrm{g}^{-1}\right)$ was observed as compared to chitosan glutaraldehyde crosslinked microspheres.
\end{abstract}

Keywords: microencapsulation; chitosan; sulfoxine.

\section{INTRODUÇÃO}

A contaminação de ambientes aquáticos com íons metálicos tóxicos é um problema complexo e a remediação desses ambientes vem recebendo atenção de muitos pesquisadores nos últimos anos. ${ }^{1} \mathrm{O}$ aumento da concentração destes metais tóxicos pode ser atribuído principalmente às intensas atividades industriais e agrícolas. Muitos destes cátions metálicos são tóxicos mesmo em baixas concentrações e deveriam ser removidos para a remediação dessas águas. ${ }^{2,3}$

O cobre é um elemento potencialmente tóxico devido ser um dos metais mais utilizados, principalmente nas indústrias elétricas, de galvanoplastia, de fertilizantes, em atividades de mineração, fundição e refino de metais, além de estar presente na composição de pigmentos, fungicidas e pesticidas. A toxicidade deste metal está relacionada com a capacidade que o elemento possui de catalisar a formação de radicais livres. De acordo com a Agência de Proteção Ambiental Americana, a concentração máxima permitida de cobre em água potável é de $1,3 \mathrm{mg} \mathrm{dm}^{-3}$. $^{2,4}$

A adsorção ou extração em fase sólida é uma técnica que vem sendo muito utilizada para a remoção de metais de águas contaminadas. Algumas das importantes vantagens da adsorção em relação às técnicas comumente usadas são: a baixa quantidade de resíduo gerado, fácil recuperação dos metais e a possibilidade de reutilização do adsorvente. ${ }^{5-7}$

O desenvolvimento de adsorventes preparados pela microencapsulação de agentes complexantes orgânicos surge como uma alternativa de grande potencial de aplicação em Química Analítica. ${ }^{8} \mathrm{O}$ uso destes adsorventes modificados com o objetivo de separar e pré-concentrar traços de íons metálicos apresenta inúmeras vantagens: o agente complexante pode ser substituído facilmente e, portanto, o mesmo adsorvente pode ser usado para diferentes propósitos. Por exemplo, um agente complexante seletivo pode ser microencapsulado, obtendo-se então seletividade

\footnotetext{
*e-mail: favere@qmc.ufsc.br
}

para um íon metálico específico; este pode ser facilmente substituído ou recuperado propiciando uma maior flexibilidade das condições de trabalho, devido o complexante não estar ligado covalentemente, o que permite ao ligante uma posição menos rígida no adsorvente. ${ }^{9,10}$

O biopolímero quitosana (QTS) tem sido considerado um promissor material polimérico e de grande interesse científico devido as suas propriedades, tais como biocompatibilidade, biodegrabilidade e não toxicidade e, é extensivamente utilizado para microencapsulação de fármacos. ${ }^{11}$

A QTS é obtida a partir da reação de desacetilação parcial da quitina em soluções alcalinas concentradas. É um copolímero constituído de unidades de $\beta(1 \rightarrow 4)$-2-amino-2-desoxi-D-glicopiranose e $\beta(1 \rightarrow 4)$-2-acetamido-2-desoxi-D-glicopiranose. A presença de grupos amino na cadeia polimérica permite que esses grupos quando protonados apresentem uma alta densidade de carga positiva propiciando a interação iônica com inúmeros agentes quelantes aniônicos. ${ }^{12-14}$

Dentre os compostos orgânicos mais conhecidos que apresentam propriedades complexantes, destacam-se as hidroxibenzopiridinas, grupo de reagentes orgânicos com alta versatilidade, podendo reagir com uma grande variedade de íons metálicos. A mais conhecida é a 8-hidroxiquinoleína (oxina). A presença de diferentes grupos funcionais, como - $\mathrm{OH},-\mathrm{COH},-\mathrm{NO},-\mathrm{SO}_{3}^{-}$, forma derivados da 8-hidroxiquinoleína. Dentre eles o grupo sulfonato $\left(-\mathrm{SO}_{3}{ }^{-}\right)$, é responsável por originar a 8-hidroxiquinoleína-5 ácido sulfônico, mais conhecido como sulfoxina (SX), que é um quelante aniônico. O nitrogênio piridínico e o grupamento fenólico presente na estrutura do composto são os responsáveis pelos processos de complexação com os íons metálicos. ${ }^{15,16}$

O objetivo do presente trabalho foi preparar microesferas de QTS reticuladas com glutaraldeído através de atomização via spray drying contendo o agente quelante sulfoxina, obtendo-se um novo adsorvente quelante (QTS-SX-Ret) a fim de avaliar suas propriedades adsortivas com relação aos íons $\mathrm{Cu}(\mathrm{II})$. 


\section{PARTE EXPERIMENTAL}

A QTS utilizada para a preparação do novo adsorvente foi fornecida pela Purifarma, Brasil, com grau de desacetilação de $90 \%$. A hidroxibenzopiridina sulfoxina utilizada era de procedência da Sigma-Aldrich, Steinheim, Alemanha. Para os ensaios de adsorção foi utilizada uma solução padrão de $\mathrm{Cu}$ (II) $10000 \mathrm{mg} \mathrm{dm}^{-3}$ da Merck. Os demais reagentes utilizados nos experimentos são de grau analítico.

\section{Preparação dos adsorventes QTS-SX-Ret e QTS-Ret via spray drying}

Uma solução de $200 \mathrm{~cm}^{3}$ de QTS 1,0\% (m/v) foi preparada com uma solução de ácido acético 5\% (v/v). Posteriormente foi adicionado o agente quelante sulfoxina na razão em massa de 1:7 (SX:QTS), mantendo a solução sob agitação até atingir a homogeneidade. A porcentagem de sulfoxina foi otimizada por meio de estudos prévios. Juntamente a essa solução foi adicionado $3,0 \mathrm{~cm}^{3}$ de agente reticulante glutaraldeído concentrado. Essa solução foi levada ao spray drying (Büchi modelo B-191). Através da atomização da solução foi obtido o adsorvente QTS-SX-Ret na forma de microesferas. Os parâmetros experimentais ajustados durante a atomização foram: temperatura de entrada do ar $180^{\circ} \mathrm{C}$, temperatura de saída $85^{\circ} \mathrm{C}$, taxa de aspiração $100 \%$, taxa de atomização $6 \%$ e fluxo de ar $500 \mathrm{NL} / \mathrm{h}$. As mesmas condições foram utilizadas na preparação da QTS reticulada (QTSRet) com glutaraldeído, sem o agente complexante SX.

\section{Espectroscopia Raman}

A espectroscopia FT-Raman foi empregada com sucesso na caracterização do agente quelante sulfoxina microencapsulado na QTS, devido à forte fluorescência da amostra. Os espectros FT-Raman foram obtidos em um equipamento Bruker RFS/100 e excitados pela linha $1064 \mathrm{~nm}$ de um laser Nd-YAG, usando $100 \mathrm{~mW}$ de potência na amostra (pó compactado) e 128 scans. A resolução espectral empregada foi $4,0 \mathrm{~cm}^{-1}$.

\section{Análise de microscopia eletrônica de varredura (MEV) e microanálise de raio-x (EDX)}

As análises das amostras de QTS-SX-Ret e QTS-Ret foram realizadas colocando as amostras sobre um suporte de latão/alumínio, e recobrindo-as com uma fina camada de ouro, e analisadas utilizando um microscópio eletrônico de varredura da marca Philips, modelo XL 30 com uma voltagem de aceleração de $20 \mathrm{Kev}$.

\section{Experimentos de adsorção}

O processo de adsorção dos íons $\mathrm{Cu}(\mathrm{II})$ em meio aquoso depende de vários fatores, como concentração do íon metálico, quantidade de adsorvente, $\mathrm{pH}$, tempo de contato e temperatura. Os experimentos foram conduzidos no sentido de avaliar a influência de alguma destas variáveis no processo de adsorção. As massas de adsorvente e as soluções de $\mathrm{Cu}(\mathrm{II})$, previamente medidas, foram colocadas em frascos fechados, mantidos em um sistema a uma temperatura de $25^{\circ} \mathrm{C}$ e agitação de 200 rpm, utilizando um equipamento Lab-Line Shaker Bath, da Marconi. Após centrifugação a 3000 rpm, as concentrações de $\mathrm{Cu}$ (II) no sobrenadante foram determinadas por espectrometria de absorção atômica em chama (F AAS), em um espectrômetro Varian, modelo SpectrAA 50 equipado com um atomizador de chama ar-acetileno e lâmpada de cátodo oco HLA-4S. Os parâmetros operacionais do F AAS foram otimizados, sendo que a taxa de aspiração usada foi de $7 \mathrm{~cm}^{3} \mathrm{~min}^{-1}$.
O estudo da dependência do $\mathrm{pH}$ na adsorção foi realizado empregando $50 \mathrm{~cm}^{3}$ de solução $20 \mathrm{mg} \mathrm{dm}^{-3}$ de $\mathrm{Cu}$ (II) e $30 \mathrm{mg}$ de adsorvente. $\mathrm{O}$ pH da solução foi ajustado com solução tampão variando de 4 a 10 e o sistema mantido sob agitação durante $24 \mathrm{~h}$. O ensaio cinético de adsorção foi realizado utilizando $200 \mathrm{~cm}^{3}$ de solução com concentração $100 \mathrm{mg} \mathrm{dm}^{-3} \mathrm{de} \mathrm{Cu}$ (II) no pH 6,0 em um frasco fechado contendo $100 \mathrm{mg}$ do adsorvente. Os experimentos de equilíbrio de adsorção foram realizados empregando $50 \mathrm{~cm}^{3}$ de solução com concentrações de $\mathrm{Cu}$ (II) variando de 10 a $400 \mathrm{mg} \mathrm{dm}^{-3}$ no $\mathrm{pH} 6,0$, num erlenmeyer fechado contendo $50 \mathrm{mg}$ do adsorvente.

\section{RESULTADOS E DISCUSSÃO}

\section{Caracterização do novo adsorvente QTS-SX-Ret}

A caracterização do novo adsorvente QTS-SX-Ret obtido por spray drying foi realizada por meio de análises de Raman, MEV e EDX. A Figura 1 representa a estrutura molecular do novo adsorvente, antes da reticulação.

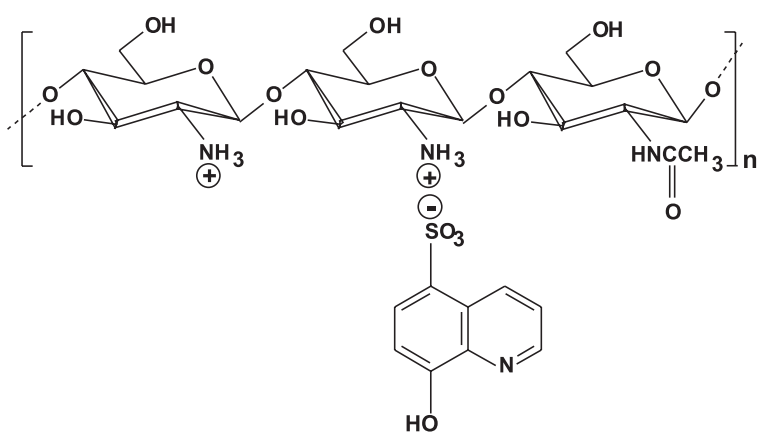

Figura 1. Estrutura molecular do novo adsorvente, antes da reticulação

As semelhanças entre os espectros Raman da QTS-Ret e QTS-SXRet, Figura 2, indicam que as bandas observadas nestes espectros são atribuídas principalmente ao reticulante. Entretanto, uma inspeção mais minuciosa no espectro da QTS-SX-Ret revela que a banda em $1367 \mathrm{~cm}^{-1}$ pode ser atribuída à molécula sulfoxina, devido à banda em $1377 \mathrm{~cm}^{-1}$ da QTS-Ret não ser tão forte como a banda em $1381 \mathrm{~cm}^{-1}$ da SX. As alterações nas posições das frequiências do Raman indicam uma efetiva interação entre a SX e a QTS. As bandas fracas em 727 e $686 \mathrm{~cm}^{-1}$, rela-

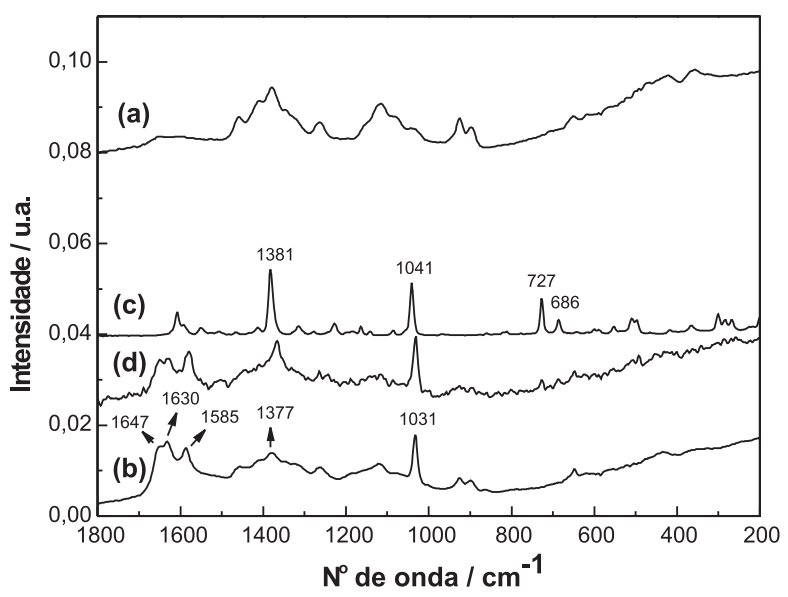

Figura 2. Espectros FT-Raman das amostras: (a) QTS, (b) QTS-Ret, (c) SX, (d) QTS-SX-Ret 
cionadas aos mesmos valores de freqüências presentes no espectro da SX, também podem ser observadas no espectro da QTS-SX-Ret, sugerindo que a microencapsulação do agente quelante no polímero ocorreu.

As análises de MEV, Figura 3, revelaram que as microesferas de QTS-SX-Ret apresentam uma superfície sem fissuras ou porosidade aparente, perfeitamente esférica, lisa e uniforme, apresentando forma semelhante às microesferas de QTS-Ret. Com relação ao tamanho das microesferas de QTS-SX-Ret obtidas, observou-se que a maioria situa entre 1,41 e $8,35 \mu \mathrm{m}$, constituindo-se em uma amostra com uniformidade similar às microesferas de QTS-Ret, as quais apresentaram tamanhos variando de 1,48 a 6,26 $\mu \mathrm{m}$. Portanto, a microencapsulação do SX na QTS não provocou alterações significativas nas características morfológicas das microesferas, quando comparadas com as microesferas de QTS reticuladas.
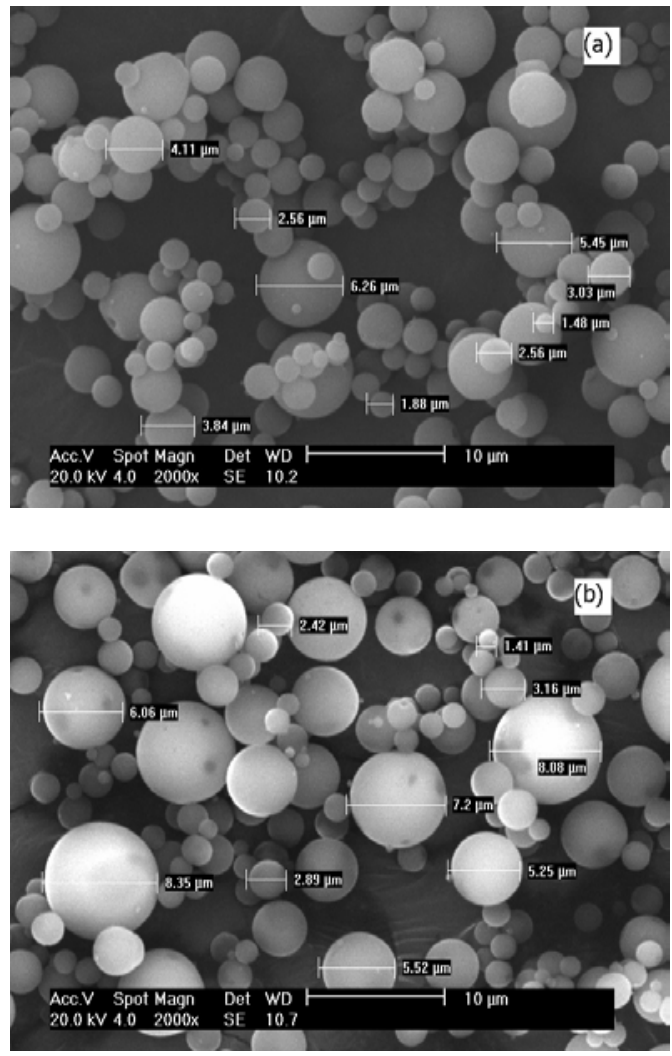

Figura 3. Microscopia eletrônica de varredura das microesferas: (a) QTSRet e (b) QTS-SX-Ret

A caracterização da QTS-SX-Ret foi realizada também empregando a técnica de análise elementar de microssonda de energia dispersiva de raio-x. A análise semiquantitativa revelou 2,36\% de enxofre no adsorvente, o qual pode ser relacionado ao grupo sulfonato da sulfoxina.

\section{Dependência do pH na adsorção}

A adsorção de íons Cu(II) pela QTS-SX-Ret, Figura 4, aumenta com o aumento do pH da solução até o valor máximo de 6,0. Em meio ácido o decréscimo da adsorção pode ser atribuído à protonação dos sítios de complexação do SX e dos grupos amino remanescentes da QTS, uma vez que o agente complexante SX apresenta duas constantes de acidez referentes ao nitrogênio piridínico e ao grupamento fenólico, $\mathrm{pK}_{1}=4,0$ e $\mathrm{pK}_{2}=8,5$, respectivamente..$^{2,17}$ Em meio muito alcalino as soluções foram tamponadas com agentes complexantes auxiliares, a fim de se evitar a precipitação dos íons $\mathrm{Cu}$ (II) na forma de hidróxidos. Porém, os compostos presentes nas soluções tampão formam complexos com o metal estudado provocando uma diminuição na adsorção dos íons pelo adsorvente o que pode justificar a diminuição da adsorção observada em pH maiores que 6,0.

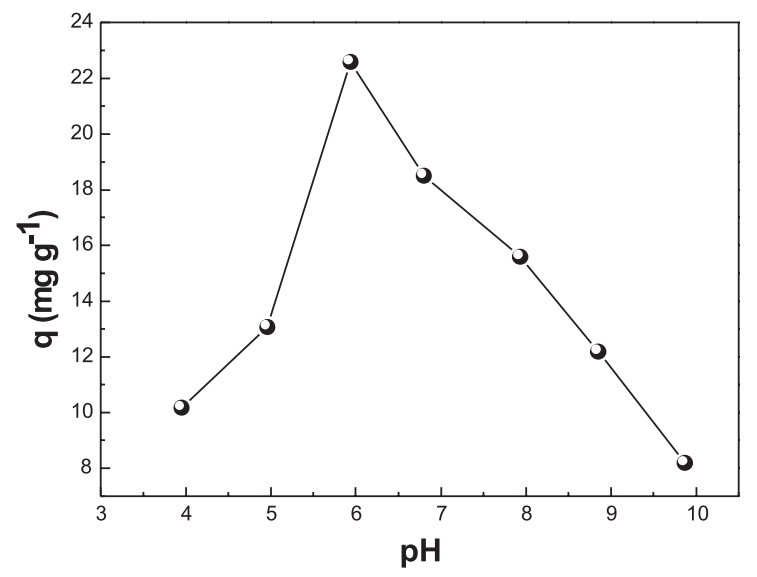

Figura 4. Quantidade de Cu(II) adsorvido pela QTS-SX-Ret em diferentes $\mathrm{pH}$ à $25{ }^{\circ} \mathrm{C}, 30 \mathrm{mg}$ de adsorvente, $50 \mathrm{~cm}^{3}$ de solução $20 \mathrm{mg} \mathrm{dm}^{-3}$ de íon metálico, tempo de contato 24 h e agitação $200 \mathrm{rpm}$

\section{Cinética de adsorção}

A Figura 5a ilustra a variação da concentração de íon metálico $\left(\mathrm{C}_{\mathrm{t}} / \mathrm{C}_{\mathrm{o}}\right)$ na solução em função do tempo de adsorção. $\mathrm{C}_{\mathrm{t}}$ e $\mathrm{C}_{\mathrm{o}}$ são as concentrações do metal no tempo te a concentração inicial, respectivamente. A curva cinética de adsorção de $\mathrm{Cu}$ (II) pela QTS-SX-Ret demonstrou que a adsorção é lenta nas primeiras horas e atinge o equilíbrio de adsorção em aproximadamente $24 \mathrm{~h}$.

Para elucidar o mecanismo cinético que rege o processo de adsorção de $\mathrm{Cu}$ (II) pela QTS-SX-Ret, foram testados os modelos cinéticos de pseudo primeira-ordem, pseudo segunda-ordem e difusão intrapartícula, sendo que a validade desses modelos pode ser interpretada pela linearidade dos gráficos $\log \left(\mathrm{q}_{\mathrm{e}}-\mathrm{q}_{\mathrm{t}}\right)$ vs. $\mathrm{t},\left(\mathrm{t} / \mathrm{q}_{\mathrm{t}}\right)$ vs. t e $\mathrm{q}_{\mathrm{t}}$ vs. $\mathrm{t}^{1 / 2}$, respectivamente. ${ }^{18-20}$

A Tabela 1 apresenta as equações lineares e os respectivos coeficientes de correlação obtidos na adsorção de $\mathrm{Cu}$ (II) pela QTS-SX-Ret. A análise dos resultados permitiu verificar que a equação de pseudo segunda-ordem, baseada na capacidade de adsorção no equilíbrio, foi a que promoveu o melhor ajuste dos dados experimentais $\left(\mathrm{R}^{2}=0,999\right)$ dentre os três modelos cinéticos testados evidenciando uma quimissorção como etapa determinante do mecanismo de adsorção e não uma transferência de massa em solução.

O tratamento dos dados cinéticos de adsorção de $\mathrm{Cu}(\mathrm{II})$ pela QTS-SX-Ret utilizando o modelo cinético de pseudo segunda-ordem,

Tabela 1. Equações lineares e respectivos coeficientes de correlação para três modelos cinéticos testados na adsorção de $\mathrm{Cu}$ (II) pela QTS-SX-Ret

\begin{tabular}{lcc}
\hline Modelo Cinético & Equação & $\mathrm{Y}=\mathrm{A}+\mathrm{BX}\left(\mathrm{R}^{2}\right)$ \\
\hline Pse. 1 ${ }^{\text {a } \text { Ordem }}$ & $\log \left(q_{e}-q_{t}\right)=\log q_{e}-\frac{k_{l}}{2,303} \cdot t$ & $\mathrm{Y}=0,824-0,001 \mathrm{X}$ \\
& $\frac{t}{(}=\frac{1}{k_{2} \cdot q_{e}^{2}}+\frac{1}{q_{e}} \cdot t$ & $\mathrm{Y}=0,396)$ \\
Pse. 2 ${ }^{\text {a } \text { Ordem }}$ & $q_{t}=k \cdot t^{1 / 2}$ & $(0,999)$ \\
Dif. Intra. & $\mathrm{Y}=28,4+0,28 \mathrm{X}$ \\
& & $(0,827)$ \\
\hline
\end{tabular}

* $\mathrm{q}_{\mathrm{t}}$ é a quantidade adsorvida no tempo $\mathrm{t}(\mathrm{min}), \mathrm{q}_{\mathrm{e}}$ a quantidade adsorvida no equilíbrio, ambas em $\mathrm{mg} \mathrm{g}^{-1}$. 
Figura $5 \mathrm{~b}$, forneceu uma constante de velocidade de adsorção $\left(k_{2}\right)$ de $1,98 \times 10^{-3} \mathrm{~g} \mathrm{mg}^{-1} \mathrm{~min}^{-1}$. A quantidade adsorvida no equilíbrio $\left(\mathrm{q}_{\mathrm{e}}\right)$ calculada a partir da equação de pseudo segunda-ordem foi $35,7 \mathrm{mg}$ $\mathrm{g}^{-1}$, a qual pode ser comparada com o valor experimental obtido de $35,6 \mathrm{mg} \mathrm{g}^{-1}$. O erro relativo determinado a partir dos valores de $\mathrm{q}_{\mathrm{e}}$ (calculado) e $\mathrm{q}_{\mathrm{e}}$ (experimental) foi menor que $0,3 \%$, confirmando que os dados experimentais se adequaram com exatidão à equação cinética de pseudo segunda-ordem.
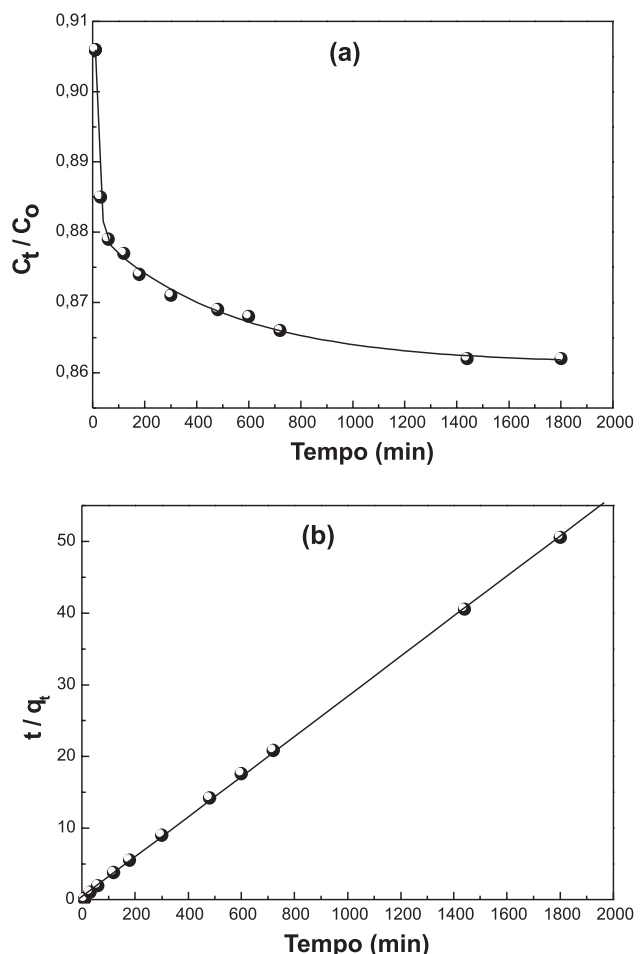

Figura 5. (a) Variação da concentração de Cu(II) adsorvido pela QTS-SX-Ret em função do tempo à $25^{\circ} \mathrm{C}, 100 \mathrm{mg}$ de adsorvente, $200 \mathrm{~cm}^{3}$ de solução de $100 \mathrm{mg} \mathrm{dm}^{-3}$ de íon metálico, pH 6,0 e agitação de $200 \mathrm{rpm}$; (b) linearização da curva cinética utilizando o modelo de pseudo segunda-ordem

\section{Isoterma de adsorção}

Os ensaios de equilíbrio foram realizados no $\mathrm{pH}$ de adsorção e tempo de contato otimizados anteriormente. Para interpretação dos dados de adsorção foi utilizado o modelo de isoterma de Langmuir, o qual considera a superfície do adsorvente como homogênea com sítios idênticos de energia. A Equação de Langmuir é representada pela Equação 1:

$\mathrm{q}=\frac{\mathrm{q}_{\mathrm{m}} \mathrm{K}_{\text {ads. }} \mathrm{C}_{\text {eq. }}}{1+\mathrm{K}_{\text {ads. }} \mathrm{C}_{\text {eq }}}$

onde, $\mathrm{q}$ é a quantidade adsorvida $\left(\mathrm{mg} \mathrm{g}^{-1}\right), \mathrm{q}_{\mathrm{m}}$ é a quantidade máxima de adsorção $\left(\mathrm{mg} \mathrm{g}^{-1}\right), \mathrm{K}_{\text {ads. }}$ é a constante de equilíbrio de adsorção e $\mathrm{C}_{\mathrm{eq}}$ é a concentração de equilíbrio $\left(\mathrm{mg} \mathrm{dm}^{-3}\right){ }^{21}$

No estudo de equilíbrio de adsorção de $\mathrm{Cu}$ (II) foram empregados como adsorventes QTS-SX-Ret e QTS-Ret, para efeito de comparação da capacidade de adsorção. Em ambos os casos, Figura 6, verifica-se a relação entre a quantidade de íon metálico adsorvido na superfície do adsorvente e a concentração deste remanescente na fase aquosa no equilíbrio. Esta relação mostrou que a capacidade de adsorção aumenta com a concentração de equilíbrio do íon $\mathrm{Cu}$ (II) na solução, alcançando progressivamente a saturação dos adsorventes testados.

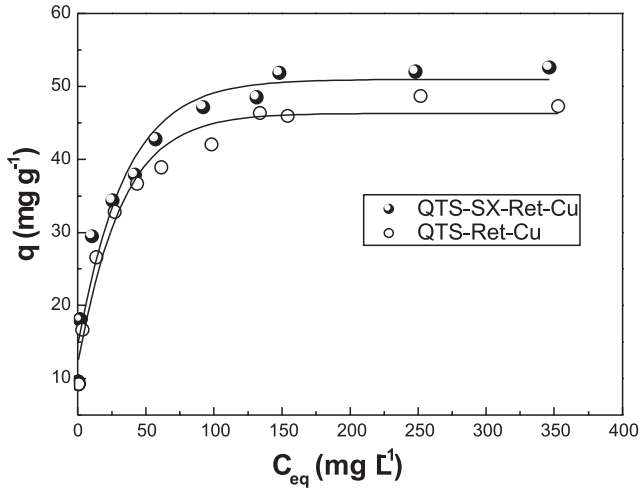

Figura 6. Quantidade de Cu(II) adsorvido pela QTS-SX-Ret e QTS-Ret, concentrações de íon metálico variando de 10 a $400 \mathrm{mg} \mathrm{dm}^{-3}$ a $25^{\circ} \mathrm{C}, 50 \mathrm{mg}$ de adsorvente, $50 \mathrm{~cm}^{3}$ de solução de íon metálico, pH 6,0, tempo de contato 24 he agitação de $200 \mathrm{rpm}$

A conversão da Equação de Langmuir para a forma linear permite calcular os valores dos parâmetros capacidade máxima de adsorção $\left(\mathrm{q}_{\mathrm{m}}\right)$ e a constante de adsorção de Langmuir $\left(\mathrm{K}_{\text {ads. }}\right)$.

A melhor regressão linear da isoterma está representada pela Equação 2:

$$
\frac{\mathrm{C}_{\mathrm{eq}}}{\mathrm{q}}=\frac{1}{\mathrm{~K}_{\mathrm{ads}} \mathrm{q}_{m}}+\frac{\mathrm{C}_{\mathrm{eq}}}{\mathrm{q}_{m}}
$$

A linearização da isoterma de adsorção, Figura 7, forneceu a equação linear: $\mathrm{Y}=0,188+0,0186 \mathrm{X}$, a qual apresentou coeficiente de correlação de 0,999 . O valor determinado da capacidade máxima de saturação da monocamada do adsorvente QTS-SX-Ret pelos íons $\mathrm{Cu}$ (II), foi 53,8 $\mathrm{mg} \mathrm{g}^{-1} \mathrm{e}$ a constante de Langmuir determinada foi $9,88 \times 10^{-2} \mathrm{dm}^{3} \mathrm{mg}^{-1}$, enquanto para a QTS-Ret se obteve a equação da reta $\mathrm{Y}=0,249+$ $0,0202 X$, com coeficiente de correlação de 0,999 . O valor determinado da capacidade máxima de adsorção de $\mathrm{Cu}$ (II) pela QTS-Ret foi de 49,5 $\mathrm{mg} \mathrm{g}^{-1} \mathrm{e}$ a constante de Langmuir foi 8,09 $\times 10^{-2} \mathrm{dm}^{3} \mathrm{mg}^{-1}$.

Comparando-se os resultados obtidos da QTS-SX-Ret em relação à QTS-Ret, observou-se um aumento na capacidade máxima de adsorção de $\mathrm{Cu}(\mathrm{II})$ de $8,0 \%$. Esse aumento observado no $\mathrm{q}_{\operatorname{maxx}}$ pode ser atribuído ao agente complexante microencapsulado na QTS, principalmente devido ao nitrogênio piridínico e ao grupamento fenólico presentes na estrutura molecular da sulfoxina. As análises dos resultados referentes aos estudos de adsorção permitiram observar que a introdução do SX na QTS provocou alterações nas propriedades adsortivas do polímero.

A literatura tem mostrado que o $\mathrm{Cu}(\mathrm{II})$ é um dos íons mais investigados em estudos de adsorção com a QTS. Nestes trabalhos verifica-se que a capacidade adsortiva desse biopolímero é fortemente influenciada por vários fatores, como grau de desacetilação, grau de reticulação e forma física utilizada. ${ }^{7,22-30}$ Neste sentido, este trabalho foi realizado apenas com a finalidade de comparar a capacidade adsortiva da QTS reticulada e da QTS reticulada contendo o agente quelante sulfoxina microencapsulado, preparados por spray drying.

\section{CONCLUSÕES}

Os estudos de caracterização utilizando as técnicas de Raman, MEV e EDX comprovaram que o agente quelante SX foi microencapsulado na superfície da QTS formando um novo material adsorvente. Verificou-se que o processo de adsorção de $\mathrm{Cu}$ (II) pela QTS-SX-Ret é dependente do pH da solução, sendo que o pH ótimo de adsorção foi 6,0. A cinética de adsorção segue o mecanismo de pseudo segunda-ordem, 


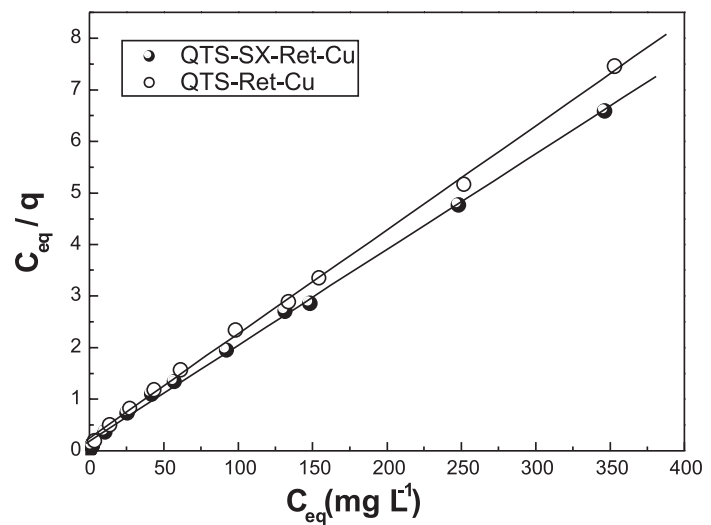

Figura 7. Linearização das isotermas de adsorção de Cu(II) pela QTS-SX-Ret e QTS-Ret, utilizando o modelo de Langmuir

modelo que promoveu a melhor correlação dos dados experimentais. As quantidades adsorvidas no equilíbrio, calculada e experimental, apresentaram uma boa concordância, desvio menor que $0,3 \%$, o que comprova a precisão do modelo cinético de pseudo segunda-ordem.

Os estudos de equilíbrio de adsorção usando a Equação de Langmuir para ajustar os dados experimentais revelaram que a capacidade máxima de adsorção da QTS-SX-Ret $\left(53,8 \mathrm{mg} \mathrm{g}^{-1}\right)$ foi maior que a da QTS-Ret (49,5 $\left.\mathrm{mg} \mathrm{g}^{-1}\right)$, aumento de cerca de $8,0 \%(\mathrm{~m} / \mathrm{m})$. Os resultados obtidos mostraram que o novo material adsorvente poderia ser testado em processos de separação e pré-concentração de íons metálicos em soluções aquosas e em águas naturais.

\section{AGRADECIMENTOS}

À Universidade Federal de Santa Catarina, ao Conselho Nacional de Desenvolvimento Científico e Tecnológico, pelo apoio financeiro, e ao LEM-IQUSP pelo uso do equipamento FT-Raman.

\section{REFERÊNCIAS}

1. Gupta, V. K.; Sharma, S.; Ind. Eng. Chem. Res. 2003, 42, 6619.

2. Justi, C. K.; Laranjeira, M. C. M.; Neves, A.; Mangrich, A. S.; Favere, V. T.; Polymer 2004, 45, 6285.

3. Akama, Y.; Yamada, K.; Itoth, O.; Anal. Chim. Acta 2003, 485, 19.

4. Galaris, D.; Evangelou, A.; Crit. Rev. Oncol. Hemat. 2002, 42, 93.
5. Pesavento, M.; Baldini, E.; Anal. Chim. Acta 1999, 389, 59.

6. Gurnani, V.; Singh, A. K.; Venkataramani, B.; Anal. Chim. Acta 2003, $485,221$.

7. Ngah, W. S. W.; Endud, C. S.; Mayanar, R.; React. Funct. Polym. 2002, 50, 181.

8. Desai, K.G. H.; Park, H. J.; J. Microencapsul. 2005, 22, 377.

9. Vitali, L.; Justi, C. K.; Laranjeira, M. C. M.; Fávere, V. T.; Polímeros: Ciência e Tecnologia 2006, 16, 116.

10. Kawamura, Y.; Mitsuhashi, M.; Tanibe, H.; Yoshida, H.; Ind. Eng. Chem. Res. 1993, 32, 386.

11. Oliveira, B. F.; Santana, M. H. A.; Ré, M. I.; Braz. J. Chem. Eng. 2005, $22,353$.

12. Kimura, I. Y.; Furlan, L.; Laranjeira, M. C. M.; Fávere, V. T.; Int. J. Polym. Mater. 2002, 51, 759.

13. Muzzarelli, R. A. A.; Chitin, $1^{\text {st }}$ ed., Pergamon Press: Oxford, 1973.

14. Rodrigues, C. A.; Laranjeira, M. C. M.; Fávere, V. T.; Polymer 1998, 39, 5121.

15. Martins, A. O.; Silva, E. L.; Carasek, E.; Gonçalves, N. S.; Laranjeira, M. C. M.; Fávere, V. T.; Anal. Chim. Acta 2004, 521, 157.

16. Martins, A. O.; Silva, E. L.; Carasek, E.; Laranjeira, M. C. M.; Fávere, V. T.; Talanta 2004, 63, 397.

17. Launay, F.; Alain, V.; Destandau, É.; Ramos, N.; Bardez, É.; Baret, P.; Pierre, J. L.; New J. Chem. 2001, 25, 1269.

18. Lagergren, S.; Handlingar 1898, 24, 1.

19. Ho, Y. S.; Mckay, G.; Process Biochem. 1999, 34, 451.

20. Weber Jr., W.; Morris, . J. J. C. ; J. Sanitary Eng. Division ASCE 1963, 89, 31.

21. Kim, C. Y.; Cho, H. T.; J. Appl. Polym. Sci. 1997, 63, 725.

22. Wu, F. C.; Tseng, R. L.; Juang, R. S.; Water Res. 2001, 35, 613.

23. Monteiro, A. C. O.; Airoldi, C.; Int. J. Biol. Macromol. 1999, 26, 119.

24. Babel, S.; Kurniawan, T. A.; J. Hazard. Mater. 2003, B 97, 219.

25. Huang, C.; Chung, Y. C.; Liou, M. R.; J. Hazard. Mater. 1996, 45, 265.

26. Lee, S. T.; Mi, F. L.; Shen, Y. J.; Shyu, S. S.; Polymer 2001, 42, 1879.

27. Kondo, K.; Nakagawa, S.; Matsumoto, M.; J. Chem. Eng. Jpn. 1997, 30, 846.

28. Wu, F. C.; Tseng, R. L.; Juang, R. S.; Ind. Eng. Chem. Res. 1999, 38, 270.

29. Wu, F. C.; Tseng, R. L.; Juang, R. S.; J. Hazard. Mater. 2000, B 73, 63.

30. Varma, A. J.; Deshpande, S. V.; Kennedy, J. F.; Carbohydr. Polym. 2004, 55,77 . 